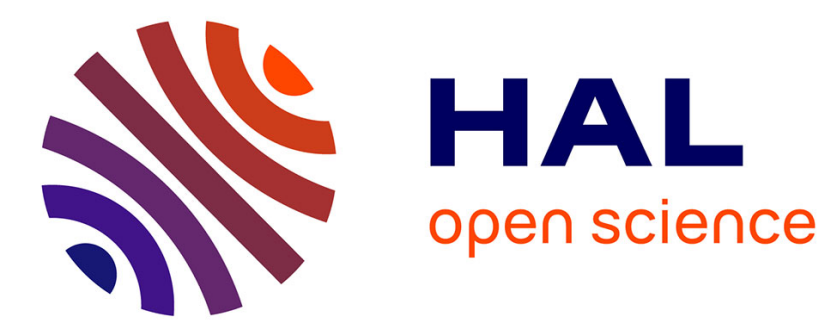

\title{
Multimodal multi-flow problem with transformation: Application to waste supply chain
}

Quentin Tonneau, Nathalie Bostel, Pierre Dejax, Romain Hospital, Valérie Mülhaupt, Thomas G. Yeung

\section{- To cite this version:}

Quentin Tonneau, Nathalie Bostel, Pierre Dejax, Romain Hospital, Valérie Mülhaupt, et al.. Multimodal multi-flow problem with transformation: Application to waste supply chain. International Conference on Industrial Engineering and Systems Management (IESM), 2015, Oct 2015, Séville, Spain. pp.876 - 882, 10.1109/IESM.2015.7380259 . hal-01621297

\section{HAL Id: hal-01621297 \\ https://hal.science/hal-01621297}

Submitted on 23 Oct 2017

HAL is a multi-disciplinary open access archive for the deposit and dissemination of scientific research documents, whether they are published or not. The documents may come from teaching and research institutions in France or abroad, or from public or private research centers.
L'archive ouverte pluridisciplinaire HAL, est destinée au dépôt et à la diffusion de documents scientifiques de niveau recherche, publiés ou non, émanant des établissements d'enseignement et de recherche français ou étrangers, des laboratoires publics ou privés. 


\title{
Multimodal multi-flow problem with transformation : application to waste supply chain
}

\author{
(presented at the $6^{\text {th }}$ IESM Conference, October 2015, Seville, Spain) (C) $\mathrm{I}^{4} \mathrm{e}^{2} 2015$
}

\author{
Quentin Tonneau* ${ }^{*}$, Nathalie Bostel ${ }^{\dagger}$, Pierre Dejax*, Romain Hospital ${ }^{\ddagger}$, Valérie Mülhaupt ${ }^{\ddagger}$ and Thomas Yeung* $^{*}$ \\ * Ecole des Mines de Nantes - IRCCyN UMR CNRS 6597, \\ 4 Rue Alfred Kastler - 44300 Nantes, France \\ \{quentin.tonneau, pierre.dejax, thomas.yeung\}@mines-nantes.fr \\ $\dagger$ Université de Nantes - IRCCyN UMR CNRS 6597, \\ 58 rue Michel Ange - BP 420 - 44606 Saint-Nazaire, France \\ nathalie.bostel@univ-nantes.fr \\ $\ddagger$ Brangeon Services, \\ 7 Route de Montjean - 49620 La Pommeraye, France \\ \{romain.hospital, valerie.mulhaupt\}@brangeon.fr
}

\begin{abstract}
This paper presents a new tactical optimization problem for non-hazardous waste and end-life product supply chain. Waste transport and recycling become crucial in our modern society, with a huge environmental and economic impact for industrials and communities. Operations on products during transport such as grinding or sorting allows companies to densify transports and reduce the overall supply cost. Integrating these new aspects, we introduce a new problem we term the multicommodity, multi-flow problem with transformations and propose a linear mathematical model to solve it. With an application to a waste transport company and a performance benchmark on a linear solver, we show the pertinence of the model in a real case study and its scalability for more complex situations.
\end{abstract}

\section{INTRODUCTION}

Transformation of products in the supply chain is very common. A product's life is composed of a large number of operations from its production (assembly of an industrial article, food industry) to its destruction (recycling, burying, energy transformation), often performed at different locations and transported in multiple ways. Numerous studies about logistics are dedicated to the production/distribution supply chain or reverse supply chain, whereas only few models are applicable to waste collection and recycling, mainly because of objective differences. A production supply chain must take into account delays and often deals with late penalties and satisfaction of customers, perishable supply chain must deliver products quickly to reduce losses. However, reprocessing supply chain objectives are cost and environmental impact reduction, regardless of delays and time to treat products.

The general problem of planing waste treatment supply chain operations can be decomposed into three major subproblems, such as long-term strategic decisions (facility location, network expansion, introduction of new technologies), tactical decisions (management of products flow, reduction of global supply chain costs), and daily operational management (routing and inventory optimization, contingency management). In this paper, we focus on the tactical part of this problem, involving product flow management, transport mode selection and transformations performed within the supply chain. This problem can be considered as an extension of the existing Multi-commodity, Multi-Modal Flow Problem, which has already been studied in the literature.

With more than 2,5 billion tons of waste produced in Europe every year, collection and transportation of household and industrial waste results in a complex and large logistic chain. We can distinguish in this domain three particular cases which are door-to-door collection, industrial and municipal waste disposal collection and hazardous waste treatment. Hazardous waste involve very specific constraints, particularly in terms of security. If hazardous waste transportation is an important research field, there are only few publications about non-hazardous waste collection and recycling supply chain, despite its importance. Studies about door-to-door collection have already been done in major cities such as Brussels [1], but industrial (and municipal disposal) waste management characteristics have not been analyzed in real case studies. A recent survey [2] proposes to split supply chain decisions for solid waste into strategic and tactical issues. The first set of problems deals with facility location such as gathering point and recycling center, and the second set is dedicated to waste flow management (within an existing network).

Focusing on tactical decisions and characteristics, there are some studies and publications approaching the subject. Guelat et al. [3] treated a multi-product multi-modal network (graph) by considering all possible transport modes for each edge and executing an extended Dijkstra algorithm on it. This method is an efficient way to determine the best path in a multi-modal graph with imposed departure and destination nodes, but is not suitable for a flow problem where outlet selection is free (or with capacity constraints). Flow splitting and merging in nodes is another difference which limits the Dijkstra algorithm to perform this task. The intermodal transportation chapter from Crainic and Kim [4] is an interesting basis to our work, but their study was focused on rail and shipping and trying to solve problems about space and infrastructure organization of docks. More recently, Chang [5] studied the MMMFPTTW (Multi Commodity MultiModal Multi Objective Flow Problem with Time Windows), which seems similar to our problem. How- 
ever, this problem has a strong set of constraints over edges, which is not the case in waste transport (where treatment and storage limits are on nodes). Lozano and Storchi [6] studied the shortest path problem in multi-modal urban network by considering "viable" paths (or sub-paths) and proposed various operations on them (concatenation, split...) . As for freight transport, priorities are not the same between passengers and waste transportation, and the definition of "viable" path doesn't occur on our study.

Considering the above aspects, we have studied a particular tactical supply chain network including transformation in hubs and described a new type of optimization problem. We proposed a mathematical linear model to solve the problem and tested it with two applications based on a waste transport company's network in order to highlight performances and applicability in a real case study.

The organization of the remainder of this paper is as follows. Section II presents a detailed description of the main problem and introduces the particular multi commodity multi-flow problem with transformations (MC-MFP-T) studied below. Section III presents the mathematical formulation of the MC-MFP-T as a linear programming model, and details the multi-modal cost selection formula. Section IV presents experimental results obtained from realistic instances and a real case study. Finally, Section V presents conclusions and directions for on-going work.

\section{PROBLEM STATEMENT}

\section{A. Nodes type and product pathway}

As presented in Fig.1, we consider a graph of four different types of nodes : Producers, Transfer Sites, Outlets and Final Customers. Producers and Customers are external companies, unlike Transfer Sites and Outlets which are internal (managed by the company) and where some decisions can be applied (transformations and reloading, see below).

Producers $(\mathrm{C} 1, \mathrm{C} 2$ and $\mathrm{C} 3$ in the figure) are the first elements of the supply chain, and represent sources of primary products. They are typically stores who evacuate their stock or more simply companies that produce waste during their operations. These products must be picked-up and treated or stored in the rest of the network. These flow of products can be routed to transfer sites to be merged and transfered to outlets via a heavier transport mode. Unlike a traditional hub network, some operations (transformations) can be performed in the transfer sites to densify or merge products. A transfer site must dispatch all incoming flows, and has a limited storage area to ensure internal logistics. Outlets are another type of internal nodes that can receive major flows of primary or pre-treated products without storage limits (like sinks in a standard flow problem). These nodes perform transformations on products, for example fine grinding of wood or repackaging of plastics, to sell final products to external customers (called final customers). Customers' requirements are fixed and a given outlet must receive enough products to fulfill the demand. We consider in this study that outlets are fixed by customers, and deliveries cannot be routed via another node. Transfer sites and outlets also produce primary products, corresponding to waste directly brought by companies or individuals in these sites. In this case, transport is not handled by the company, but flows enter in the supply chain. Note that these production quantities are similar to a traditional (external) producer.

\section{B. Products and transport compatibilities}

We must consider in this problem that any physical difference (density, packaging) leads to different products. For example, raw and ground wood are treated as two distinct products. This distinction allows us to decompose a product transformation (or recycling) into intermediate steps and states, and do preliminary transformations in transfer sites (during the supply). Final customers can express precise product specifications without being mingled with a similar existing flow. Multiple transport modes are used in the network, and the choice of them for a given edge must consider the following compatibility constraints :

- Each node is associated with a list of compatible transport modes. As all sites are different, some of them don't have infrastructure or equipment to handle all transport modes (for example automatic pick-up tailors).

- Each transport mode and node can only handle a restricted list of products. For the same reason as above, some specific products (for example liquids) cannot be routed to non equipped (or authorized) sites. Outlets are specific and only treat a limited number of product categories.

\section{Demands}

We consider in this problem two types of demands:

- Pickup demands : producers' waste which have to be picked-up without imposed route or destination. The objective for this demand is only to collect and store / treat the product along the network (transfer sites, outlets or final customers).

- Delivery demands : products that must be delivered from a fixed node to another, without any transformation, transfer or merge.

Delivery demands can be expressed by customers ordering products from a particular site ( $D 2$ in the figure), but also by producers which have particular requirements and want their products to be delivered to a particular customer or outlet (like the demand $D 1$ in the figure 1).

The second type of demand is very simple to handle because origins and destinations are fixed and the only remaining decision is the transport mode selection. But as seen in figure 1 , outlet $O 2$ must be sufficiently supplied in product $p 2$ to transform it into $p 3$ and meet the need of customer $f 1$. These deliveries can't be ruled out of the problem because even if associated decisions are simple, they have significant impacts on the rest of the network.

\section{Transformations within nodes}

Transformations denote all possible operations on products along the supply chain. They can change a product state (grinding wood for example), split a flow (sorting of various products in a flow), or merge them (assembly or composition). 
An operation is characterized by incoming products, outgoing products (defined by a kind of "recipe") and a unit cost. Each transfer node and outlet can perform a transformation up to a limit (depending on node and transformation type). Transfer nodes will typically perform minor preliminary operations like sorting, precrushing, conditioning (to transfer via heavy transport modes), while outlets treat and transform primary products into final products (resold). Depending on infrastructures and materials, some sites are not able to perform operations. In this case, we fix the limit to zero. Table I shows a possible representation of various transformations. Each transformation (row) is composed of a set of negative numbers for incoming products (consumed) and positive numbers for outgoing products (results). In this example, transformation $T 1$ transforms a ton of product $p 2$ into a ton of $p 3$ at a cost of $10 €$. This basic operation can be used to simulate packaging, grinding or compacting. T2 could represent a separation (sorting a mixed flow into two products for example), T3 an assembly or mixture of products. Transformation $T 4$ is a destructive operation, for example when a product is treated by an external provider. This operation allow transfer sites to treat or evacuate products without routing them to an outlet, but generally at a high cost. Transformation $T 5$ can be assimilated to a production of a shared resource such as manpower. This resource (modeled as a product) is consumed by multiple operations and limiting the transformation $T 5$ leads to a global (and shared) transformation quota in the node. The example in Table I represents a manpower cost per hour, and negative elements in the associated column indicate time consumption per unit operation.

\section{E. Objective and expected decisions}

The objective of this problem is to satisfy all demands with a minimal transportation and transformation cost over a single period of time. Transportation costs include the routing costs and all involved logistic costs, like pickup and unloading of each transport, transfer and temporary storage cost. An important part of cost reduction is due to transfer sites and available transformations into them. If major external producers don't produce enough goods to deploy a fleet of heavy vehicles (tractor-trailer), we should reduce the distance of light transport modes and merge all these flows in transfer sites to reduce the total supply cost. By performing some operations at these nodes, a lot of products can be densified (crushed, compacted) or directly routed (after sorting or cleaning operation) to an appropriate outlet, which further reduces the global routing cost.

The solution in Figure 1 illustrates the problem and its solution. Production of $P 2$ from $C 2$ and $C 3$ must be routed to the outlet $O 2$ to satisfy the demand $D 2$ of the final customer. These flows are merged in transfer site 1 to reduce transportation cost. Since transport mode $M 2$ is cheaper than $M 1$, we route product $P 2$ from $C 3$ through the transfer site 2 to massify the transport. Demand $D 1$ fixed the outlet for product $P 2$ incoming from $C 1$, and the only compatible transport mode between these two nodes is $M 1$. Product $P 1$ doesn't have imposed outlet, and the product is routed to a compatible one (O3). Note that modes $M 1$ and $M 2$ are compatible with $P 1$, but $O 3$ is only compatible with $M 1$.

As we have seen, this problem is an extension of the

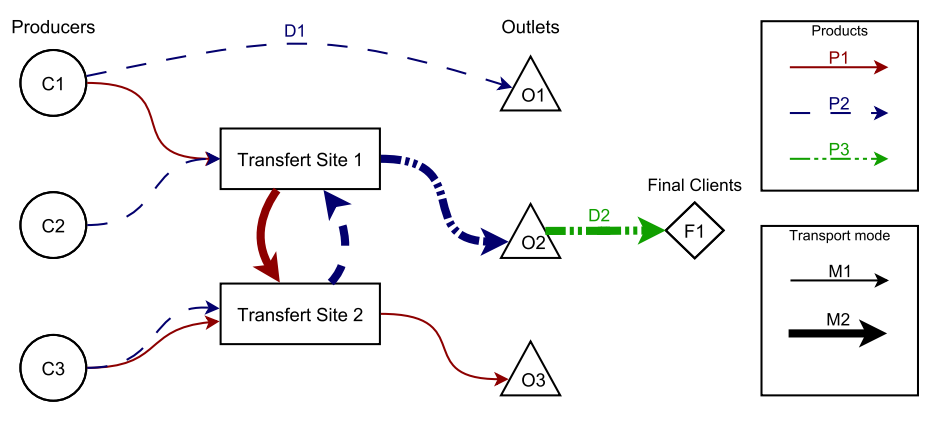

Fig. 1. Example of a MC-MMFP-T solution

TABLE I. POSSIBLE REPRESENTATION OF TRANSFORMATIONS

\begin{tabular}{l|l|l|l|l|l|l} 
Name & $\mathrm{p} 1$ & $\mathrm{p} 2$ & $\mathrm{p} 3$ & $\mathrm{p} 4$ & manpower & cost \\
\hline \hline T1 & 0 & -1 & 1 & 0 & -0.3 & 10 \\
T2 & -1 & 0.7 & 0.3 & 0 & -1 & 17 \\
T3 & 0 & -1 & -1 & 1.7 & -0.5 & 2 \\
T4 & -1 & 0 & 0 & 0 & 0 & 55 \\
T5 & 0 & 0 & 0 & 0 & 1 & 30 \\
\hline
\end{tabular}

original Min Cost Flow Problem, involving a multi-modal network, and a transformation layer which links products together and prevents us to split the problem. This augmented flow problem can be called Minimum Cost Multi-Modal Flow Problem with Transformations (abv. MC-MMFP-T), as the multi-products part becomes implicit if a transformation exists. According to the classification proposed by Ghiani et al. [2], this new problem is a $1 / S, P /$ multiwaste/TC, $P C$, but based on a flow problem rather than a location (and assignement) problem.

\section{MODEL FORMULATION}

The model presented in this section is an evolution of a standard min cost flow problem introduced by Klein in 1967 [7].

\section{A. Multi-modality and transport cost}

At this level of decision, we consider each transport mode associated with an unlimited number of vehicles. This assumption avoids daily transportation organization, and we can select the costless transport mode for each edge before starting to solve the problem. We therefore treat the multi-modal part of the problem as a pretreatment solution phase. Freight transportation costs are mainly based on time and distance. A road network company will take into account the distance to calculate petroleum consumption, vehicle maintenance cost, tire wear. The pickup and unloading times are added to the travel time to determine approximately the driver and logistic operations cost.

We choose to define edges with three indexes $(i, j, p)$ which correspond to the origin node, the destination node and the product. Each edge is associated to a cost in $€ / t$. To calculate an edge cost for a given transport mode $m \in M$, we use the following components :

- $\quad$ A distance cost $C_{d}^{m}$ (in $\left.€ / \mathrm{km}\right)$

- A time cost $C_{t}^{m}$ (in $€ / \mathrm{h}$ )

- $\quad$ A pickup $T p^{m}$ and a delivery $T d^{m}$ time (in hours) 
- A volume capacity $V \max ^{m}$ (in $\mathrm{m}^{3}$ )

- A weight capacity $W \max ^{m}$ (in t)

- $\quad$ A speed $v^{m}$ (in $\mathrm{km} / \mathrm{h}$ )

For a given distance $d_{i, j}$ (in $\mathrm{km}$ ) and a product $p$ of density $\rho^{p}$ (in $\mathrm{t} / \mathrm{m}^{3}$ ), we can determinate the best transport mode and its $\operatorname{cost} c_{i, j}^{p}$ with

$$
c_{i, j}^{p}=\min _{m \in M}\left\{\frac{C_{k}^{m} d_{i, j}+\left(T p^{m}+T d^{m}+\frac{d_{i, j}}{v^{m}}\right) C_{h}^{m}}{\min \left(W \max ^{m}, \rho^{p} \times V \max ^{m}\right)}\right\}
$$

where $M$ is the set of common compatible transports between nodes $i, j$ and the product $p$. This formula calculates for each transport mode $m$ the travel cost (time cost, distance cost) and divide it by the filling limit (minimum between volume and weight limit). Then, the cheapest transport mode is chosen for the arc $(i, j, p)$. This pretreatment on the graph defines the best transportation mode for each edge in the graph. Thus we can remove the multi-modal layer from the original problem and keep only three indexes for edges, variables and parameters.

\section{B. The model}

After removing transport mode layer, the transport network is represented by a directed graph $(N, E)$ where each edge is characterized by a source, a destination and a product. Thus, we associate a unit $\operatorname{cost} c_{i, j}^{p}$ per ton of product to each edge $(i, j, p)$. We first define some notations.

\section{Domains / Sets}

$\begin{array}{ll}P & \text { Products } \\ T & \text { Transformations } \\ N & \text { Nodes } \\ A \subseteq N & \text { Producers (sources) } \\ B \subseteq N & \text { Transfer sites } \\ C \subseteq N & \text { Outlets (sink) } \\ D \subseteq N & \text { Customers (of final products) } \\ E & \text { Edges }\end{array}$

\section{Parameters (all in $\mathbb{R}^{+}$)}

$q_{i}^{p} \quad$ quantity of product $p$ produced by node $i$ $l_{i, j}^{p} \quad$ delivery demand of $p$ from node $i$ to node $j$ $c_{i, j}^{p_{j}} \quad$ transport cost of edge $(i, j, p)$

$\nu_{p}^{t} \quad$ coefficient of product $p$ in transformation $t$

$c_{i}^{t} \quad$ unit cost of transformation $t$ in node $i$

$y_{\max }{ }_{i} \quad$ capacity of transformation $t$ in node $i$

$b_{i}^{p} \quad$ storage capacity of product $p$ in node $i$

Variables

$\begin{array}{ll}x_{i, j}^{p} & \text { flow quantity of product } p \text { through arc }(i, j, p) \\ y_{i}^{t} & \text { quantity of transformation } t \text { at node } i\end{array}$

Storage at transfer nodes is limited and capacities are much lower than the amount of products that pass annually through them. Therefore, most of incoming products are transformed or routed to an outlet.

The model which we propose is as follows
Minimize

$$
\sum_{(i, j, p) \in E} c_{i, j}^{p} x_{i, j}^{p}+\sum_{t \in T} \sum_{i \in B \cup C} c_{i}^{t} y_{i}^{t}
$$

Subject To

$$
\begin{array}{ll}
\sum_{(i, j, p) \in E}\left(x_{i, j}^{p}-l_{i, j}^{p}\right)=q_{i}^{p} & \forall i \in A, \forall p \in P \\
x_{i, j}^{p} \geq l_{i, j}^{p} & \\
q_{i}^{p}+\sum_{(j, i, p) \in E} x_{j, i}^{p}-\sum_{(i, j, p) \in E} x_{i, j}^{p} & +\sum_{t \in T} y_{i}^{t} \nu_{p}^{t} \geq 0 \\
& \forall i \in B \cup C, \forall p \in P \\
q_{i}^{p}+\sum_{(j, i, p) \in E} x_{j, i}^{p}-\sum_{(i, j, p) \in E} x_{i, j}^{p} & +\sum_{t \in T} y_{i}^{t} \nu_{p}^{t} \leq b_{i}^{p} \\
& \forall i \in B, \forall p \in P \\
y_{i}^{t} \leq y_{\max i}^{t} & \forall i \in B \cup C, \forall t \in T \\
x_{i, j}^{p} \in \mathbb{R}^{+} & \forall(i, j, p) \in E \\
y_{i}^{t} \in \mathbb{R}^{+} & \forall i \in B \cup C, \forall t \in T
\end{array}
$$

The objective function (1) is composed of transport costs and transformation costs. (2) force the solution to collect all products at producers' sites. Note that delivery requests $l$ are not included in production $q$ in our formulation. Removing the $l_{i, j}^{p}$ components of the left side of (2) will count these shippings as part of the production. Constraints (3) ensure deliveries of products to final customers (fixed quantities and origins). Equations (4) and (5) define storage and transit rules at transfer nodes. The first set of constraints (4) ensures that any outgoing flow of an outlet must be supplied by incoming flows, transformations or self production. The second set (5) is similar, but limits the positive balance between incoming and outgoing flows for each product with a parameter $b$ in transfer nodes. Note that these constraints do not limit the quantity of products passing through transfer sites, but only storage at the end of the period (balance). Finally, (6) represent transformation capacity $y_{\max }$ on transfer nodes and outlets.

\section{EXPERIMENTS AND APPLICATIONS}

We tested the relevancy of our proposed model using two different applications: a real case study in a French waste transport company and a realistic benchmark of thirty generated instances coming from the first case. We considered in each case, three different transport modes, which are dump trucks, trailer dump trucks (able to transport two dumps at the same time) and semi-trailers for enlarged transports. If the number of transport modes does not impact the model complexity (because this layer is considered as a pretreatment), transport cost ratio between internal nodes and external ones must be realistic to ensure the solution quality. All experiments and measures are based on a 32 bit Linux 3.13 server with a $4 \times 2,33 \mathrm{GHz}$ processor and $16 \mathrm{~Gb}$ memory. The problem generator was implemented in a NodeJS app (v 0.12.0) and the resulting problem (in MPS format) is solved by both Coin LP solver (1.15.5) and the CPLEX (12.6.1) commercial solver. Since their performance measures are almost identical in our 
TABLE II. TRANSFORMATIONS IN THE REAL CASE APPLICATION

\begin{tabular}{c|c|c|c|c} 
Name & $\mathrm{A}$ & $\mathrm{B}$ & $\mathrm{C}$ & Unit Cost \\
\hline \hline$t 1$ & -1 & 1 & 0 & $c 1$ \\
$t 2$ & 0 & -1 & -1 & $c 2$ \\
\hline
\end{tabular}

benchmarks, we only provide results coming from the CLP solver.

\section{A. Real case application}

To ensure the realism and utility of our approach, we tested the model presented in section III in a real case situation coming from a French transport and waste treatment company named Brangeon.

Collection and transport of industrial and collective (via municipal waste disposals) waste is a very important activity of the group, and its network structure is very similar to the problem described in section II. We studied the case of a subset of waste collected and treated for an entire year. Wood (waste) collection and management system features all characteristics of the MC-MMFP-T problem, with various producers (from small waste disposal to big companies), transformations (slow and quick grinding, sorting of different types), transfer sites (grinding platforms or dedicated waste disposal), outlets (for precise wood packing and storage) and external customers (manufacturers of particle boards or firewood for example).

We considered in this application three different wood types denoted $A, B$ and $C$. Producers mainly provide wood of type $A$, and rarely of type $B$. Wood $B$ is an intermediate state of transformation, denser than $A$ but not yet ready for resale. Wood $C$ is the final state of treated wood, and represents the main part of deliveries to final customers. Two transformations $t 1$ and $t 2$ can transform wood $A$ to $B$ and $B$ to $C$ as explained in table II. Transformation $t 1$ can be performed in several transfer sites and all outlets as a pretreatment of wood. Transformation $t 2$ is reserved for outlets, to deliver customers in product $C$. Two transport modes $M 1$ and $M 2$ are considered. Producers, transfer sites and outlets are mainly compatible with the first transport mode (light dump truck). The second transport mode $M 2$ is heavier (and cheaper for long range) and mainly used to link all internal nodes and final customers.

The described problem is composed of :

- $\quad$ about 1000 producers,

- 11 transfer sites,

- 2 outlets,

- about 50 external customers,

- about forty thousand tons of wood collected and ten thousand tons of wood shipped to customers,

- 10000 edges in the graph.

We obtained an initial simplex matrix of about 10000 columns and 5500 rows, solved in $50 \mathrm{~ms}$ with CLP on the computer described in section IV. We compared the given solution with real decisions and costs involved during the period studied. Results are shown in table III. The studied network is already "manually" optimized by the company,
TABLE III. MODEL IMPROVEMENTS IN REAL SITUATION

\begin{tabular}{l|l}
\multicolumn{1}{c}{ Indicator } & \multicolumn{1}{c}{ Variation } \\
\hline Product flows routed via a transfer node & $+7 \%$ \\
Ratio of transformed products in transfer nodes & $+23 \%$ \\
Collection cost & $-17.51 \%$ \\
Transformation cost & $+20.11 \%$ \\
Transfer cost & $-5.54 \%$ \\
\hline Total & $-14.72 \%$ \\
Total (including imposed deliveries cost) & $-10.71 \%$
\end{tabular}

which handle $31 \%$ of products coming from producers through transfer sites. The choice of transport modes is similar both in the solution and real situation, indeed this part of the problem is already optimal.

The optimal solution increases the quantity of initial products passing through transfer sites to $38 \%$ and maximize the usage of the transformation $t 1$. The ratio of product transformed before being transfered to an outlet jumps from $55 \%$ to $78 \%$, which shows transformation relevancy in the supply chain. As a consequence, the collection cost (from producers to internal network) is reduced and light trucks are less used. The distance of the local loop (collection network) is reduced, which could improve the quality of service and the reactivity (if a new market appears). The transformation cost increases proportionally to its use $(+20 \%)$ but this surplus is offset by the gain of internal transportation cost. Indeed, the optimal solution increases internal transit by $7 \%$ (in tons) while reducing this part of the total cost by $5 \%$. This gain is due to transformations which increase the product density and reduce the number of necessary round-trips. Overall, the total supply chain cost from producers to outlets is reduced by $14.7 \%$, and the total supply cost for the company (including imposed deliveries where no optimization are possible) is reduced by $10.71 \%$. Considering that this network has already been manually optimized, we obtain huge savings based on a better network organization. This conclusion encourages us to apply this method for other product types, especially for those that don't already exploit transfers and transformations.

\section{B. Realistic benchmark}

To benchmark the model on major problems, involving hundreds of products and thousands of nodes in the graph, we generated a set of instances coming from the case study above and studied the generation and solution time and the simplex matrix characteristics, to determinate both the potential and limits of this approach with solvers (free and commercial).

\section{1) Generated instances:}

We generated thirty different instances (1-30) coming from the previous study by randomly adding or removing nodes (and associated productions) in the network. Transport costs are coming from the $\mathrm{CNR}^{1}$, a French organism responsible of collecting and analyzing road transport cost calculation. Products densities are picked-up from $\mathrm{ADEME}^{2}$. These sources ensure a realist but simplified case study to evaluate model performances. All instances have the following similar characteristics :

\section{- 275 products,}

\footnotetext{
${ }^{1}$ Comité National Routier

${ }^{2}$ Agence de l'Environnement et de la Maîtrise de l'Energie
} 
TABLE IV. REALISTIC INSTANCE SET

\begin{tabular}{|c|c|c|}
\hline Name & Number of Nodes & Number of edges \\
\hline 1 & 100 & 274720 \\
2 & 200 & 297825 \\
3 & 300 & 379288 \\
4 & 400 & 448961 \\
5 & 500 & 500855 \\
6 & 600 & 605595 \\
7 & 700 & 665433 \\
8 & 800 & 757470 \\
9 & 900 & 824436 \\
10 & 1000 & 893333 \\
11 & 1100 & 1065203 \\
12 & 1200 & 1252512 \\
13 & 1300 & 1415011 \\
14 & 1400 & 1587641 \\
15 & 1500 & 1769655 \\
16 & 1600 & 1962752 \\
17 & 1700 & 2119614 \\
18 & 1800 & 2280053 \\
19 & 1900 & 2603302 \\
20 & 2000 & 2781073 \\
21 & 2100 & 3022452 \\
22 & 2200 & 3096814 \\
23 & 2300 & 3290302 \\
24 & 2400 & 3490506 \\
25 & 2500 & 3695072 \\
26 & 2600 & 3906134 \\
27 & 2700 & 3924673 \\
28 & 2800 & 4211490 \\
29 & 2900 & 4505778 \\
30 & 3000 & 4961234 \\
\hline
\end{tabular}

- 30 transformations,

- 3 transport modes.

All instances are detailed in table IV, with the number of nodes and edges in the graph. We observe that the number of edges in the graph increases polynomially with respect to the number of nodes.

\section{2) Results:}

Measures and results coming from instances 1 to 30 are indicated in Table $\mathrm{V}$. We indicate for each instance the number of columns, rows and non-zero elements of the initial simplex matrix (before pre-solving), the time $\left(G_{\text {time }}\right)$ required to generate the problem (in MPS format), the solving time $S_{\text {time }}$ and the number of simplex iterations (Iter) to reach the optimal value with the solver, and the number of active (nonzero) variables $k$ in the optimal solution. We tried to generate instances with more than 3000 nodes, but these problems could not be solved either by CPLEX or by CLP (these two solvers with standard configuration encounter memory allocation limits). Figure 2 shows generation time, solving time and the number of edges depending on the number of nodes.

We observe in this first benchmark that the problem is easy to solve (about 40 seconds for a 3000 nodes graph). Generation of the instance in MPS format is longer than the solving time, which indicates that the pretreatment of the problem is very long. Regarding the algorithm complexity, we observe that the generation time is upscaled by the number of edges (we need to enumerate all edges in the model, and choose for each one a transport mode, respecting compatibilities). We also observe that the solving complexity (time or iterations) is highly correlated with the number of edges, which is exponential on graph size (number of nodes). As products can be routed through every transfer nodes and outlets, we produce at least $(|A|+|B|+|C|) \times(|B|+|C|)$ edges if producers only emit one product.
TABLE V. BENCHMARK RESULTS

\begin{tabular}{|c|c|c|c|c|c|c|c|}
\hline Name & Row & Columns & Edges & $G_{\text {time }}$ & $S_{\text {time }}$ & Iter & $\mathrm{k}$ \\
\hline 1 & 27400 & 274750 & 591818 & 2.689 & 1.152 & 189 & 93 \\
2 & 54800 & 297855 & 657249 & 2.954 & 1.272 & 664 & 259 \\
3 & 82748 & 379318 & 907600 & 3.752 & 2.232 & 1183 & 392 \\
4 & 110696 & 448991 & 1125241 & 4.647 & 2.762 & 1822 & 547 \\
5 & 137274 & 500885 & 1287836 & 5.214 & 3.092 & 2420 & 704 \\
6 & 164126 & 605625 & 1627328 & 6.333 & 3.832 & 3048 & 850 \\
7 & 190978 & 665463 & 1820418 & 6.958 & 4.252 & 3718 & 1004 \\
8 & 218378 & 757500 & 2122738 & 7.979 & 5.012 & 4627 & 1181 \\
9 & 245230 & 824466 & 2342544 & 8.691 & 5.452 & 5442 & 1373 \\
10 & 272356 & 893363 & 2570817 & 9.53 & 5.992 & 5878 & 1519 \\
11 & 300578 & 1065233 & 3156177 & 11.4 & 7.272 & 6841 & 1686 \\
12 & 328800 & 1252542 & 3801398 & 13.456 & 9.022 & 7626 & 1837 \\
13 & 356200 & 1415041 & 4364641 & 15.473 & 10.092 & 8511 & 1988 \\
14 & 383326 & 1587671 & 4966943 & 17.355 & 11.512 & 9403 & 2145 \\
15 & 411000 & 1769685 & 5606382 & 19.529 & 13.172 & 10206 & 2290 \\
16 & 438400 & 1962782 & 6287395 & 21.659 & 14.832 & 11199 & 2443 \\
17 & 465526 & 2119644 & 6838068 & 23.418 & 16.242 & 12093 & 2625 \\
18 & 492926 & 2280083 & 7405241 & 25.29 & 17.572 & 12807 & 2787 \\
19 & 521970 & 2603332 & 8566391 & 29.062 & 19.972 & 13906 & 2932 \\
20 & 549096 & 2781103 & 9200264 & 30.581 & 21.862 & 14703 & 3091 \\
21 & 576770 & 3022482 & 10067587 & 33.607 & 23.732 & 15785 & 3259 \\
22 & 603348 & 3096844 & 10324141 & 34.637 & 24.112 & 16461 & 3424 \\
23 & 631570 & 3290332 & 11018352 & 37.739 & 26.162 & 17363 & 3587 \\
24 & 658422 & 3490536 & 11737396 & 39.25 & 29.322 & 18799 & 3755 \\
25 & 685822 & 3695102 & 12474882 & 41.483 & 31.132 & 19903 & 3910 \\
26 & 713770 & 3906164 & 13236610 & 43.788 & 31.542 & 20755 & 4069 \\
27 & 740622 & 3924703 & 13287299 & 43.552 & 31.792 & 21690 & 4240 \\
28 & 767474 & 4211520 & 14327926 & 47.057 & 33.972 & 22668 & 4426 \\
29 & 793504 & 4505808 & 15400363 & 50.494 & 36.762 & 23597 & 4598 \\
30 & 822548 & 4961264 & 17068662 & 54.607 & 40.592 & 25033 & 4800 \\
\hline
\end{tabular}

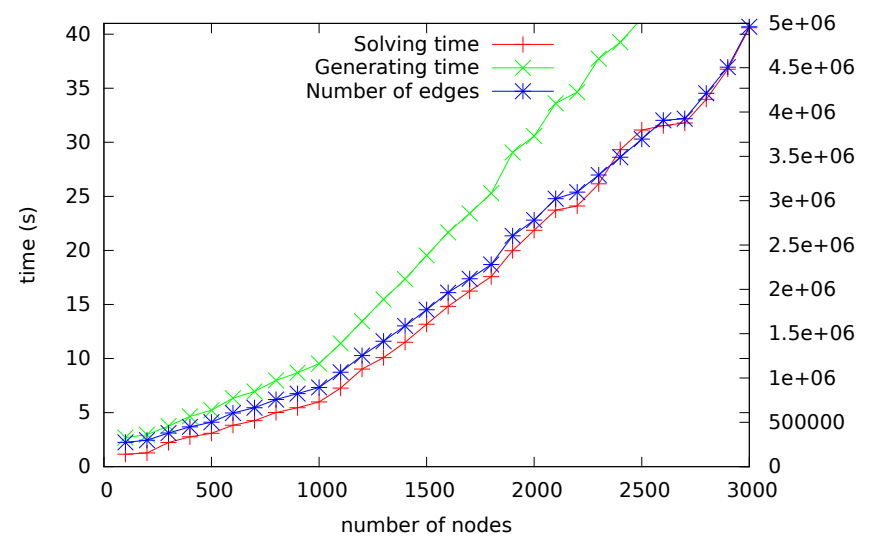

Fig. 2. First Benchmark

If the number of edges in the graph is very large compared to the number of nodes, they are rarely used in the optimal solution. In all generated instances, less than $0.2 \%$ of initial variables (mainly composed of edges) are used in the optimal solution. The result graph of the larger instance (30) is fifty times smaller than the initial graph of instance 1 (solved in 1s). Significant work could be done to efficiently detect and remove unused edges without compromising the optimality of solutions, greatly improving the model performance and overcoming the limits encountered with CPLEX and CLP.

In a real industrial context, tactical decisions applied to this kind of problem are taken for long run periods (one year). The proposed model applied to a 3000 nodes graph with more than 250 products should cover all requirements of a large waste collection company, and return optimal solutions in reasonable time (less than five minutes). 


\section{CONCLUSION}

In this paper we have presented a mathematical formulation for the Multi Commodity Multi-Flow Problem with Transformations over a single period. This is a new tactical optimization problem designed from waste and end-life product supply chain. The objective is to improve the overall supply chain cost by allowing transformations in transfer sites to enlarge and merge product flows and reduce the transport cost. The proposed model has been illustrated through a real case study in a waste collection company, and extended to a realistic benchmark to identify performances and limits on large situations. We have shown through two applications that the proposed model is applicable in a real situation, and can be theoretically applied for larger cases in reasonable time (less than one minute). By significantly reducing the supply chain cost for an already "manually" optimized situation, we demonstrated the relevance of the model for a waste collection and recycling company.

The number of edges in the model is the main factor of performance limit, and filtering useless edges during generation without compromising the optimality of solutions is a promising way to improve performances on linear solver. The implementation of the model in a real company (Brangeon) showed that new sets of constraints could improve the practicality of the model and applicability of decisions in a daily operational context :

- Transport and operation costs are partially composed of fixed costs such as deployment of a mobile crusher or packer. Renting of materials also implies a fixed cost (per day). Introducing these new elements would change the model into a mixed integer linear problem, and greatly increase the complexity to solve it.

- The current model can restrict the use of transformations and storage in transfer sites, but does not limit the quantity of products transiting through it. A new set of constraints limiting transfers into nodes should improve realism by introducing legal authorization volume for each site.
- Tactical decisions are made for a medium term period (one year) and have to be robust for minor changes. A decision made for an entire year should be daily viable and turning the model into a muti-period one to take into account seasonality of demands could improve applicability of solutions. Again, this change will increase the model complexity and new solving methods would have to be implemented.

This paper focuses on the tactical part of the non-hazardous waste management. The two other decisions categories (strategic and operational) should be studied and used in coordination with it to fully optimize the supply chain, by improving the network structure (new facility location, technology deployment, commercial strategies) and optimizing the daily vehicle routing. In the future, we plan to work along these guidelines.

\section{REFERENCES}

[1] T. Kulcar, "Optimizing solid waste collection in Brussels," European Journal of Operational Research, vol. 90, no. 1, pp. 71-77, 1996.

[2] G. Ghiani, D. Laganà, E. Manni, R. Musmanno, and D. Vigo, "Operations research in solid waste management: A survey of strategic and tactical issues," Computers \& Operations Research, vol. 44, pp. 22 - 32, 2014.

[3] J. Guelat, M. Florian, and T. Crainic, "A multimode multiproduct network assignment model for strategic planning of freight flows," Transportation Science, vol. 30, no. 3, pp. 231-250, 1990.

[4] T. Crainic and K. Kim, "Intermodal transportation," Transportation, vol. 64, no. 6, pp. 15-18, 2006.

[5] T. Chang, "Best routes selection in international intermodal networks," Computers \& Operations Research, vol. 35, no. 9, pp. 2877-2891, 2008.

[6] A. Lozano and G. Storchi, "Shortest viable path algorithm in multimodal networks," Transportation Research Part A: Policy and Practice, vol. 35, no. 3, pp. 225-241, 2001.

[7] M. Klein, "A Primal Method for Minimal Cost Flows with Applications to the Assignment and Transportation Problems," Management Science, vol. 14, pp. 205-220, 1967. 